ISSN: 0212-0267

DOI: http://dx.doi.org/ro.I420I/hedu20I837365382

\title{
LOS PLANES DE ESTUDIOS DE LA ESCUELA NORMAL DEL MAGISTERIO PRIMARIO DE CÁCERES EN LA SEGUNDA REPÚBLICA
}

\section{The curriculum of the Normal School of Primary Education of Cáceres in the Second Republic}

\author{
Francisco Javier Alejo Montes \\ Universidad de Extremadura \\ Correo-e: alejo@unex.es
}

Recepción: I9 de diciembre de 20I7. Envío a informantes: 29 de enero de 2018 Aceptación definitiva: 3 de marzo de 2018

Resumen: En este trabajo se analizan los distintos planes de estudio que convivieron en la Escuela Normal del Magisterio Primario de Cáceres durante la Segunda República, desde el Plan Bergamín de 19i4, en el que continuaron aquellos alumnos y alumnas que ya habían comenzado sus estudios en años anteriores, y a los que se les dio la posibilidad de ingresar en el cuerpo del magisterio mediante su participación en los Cursillos de selección profesional; siguiendo con el Plan preparatorio, para aquellos a los que les sorprendió la transición de planes de estudio, pero no tenían el título de bachiller superior exigido en el nuevo, y finalizando con el plan estrella de la Segunda República, el denominado Plan Profesional. En ellos se analizan los cursos impartidos, los contenidos, la reválida final de estudios, los tribunales de exámenes y las formas de evaluar en la Escuela Normal cacereña.

Palabras Clave: currículum, formación de docentes de primaria, Historia de la Educación, Segunda República, España.

AвSTRACT: In this paper we analyze the different curricula that coexisted in the Normal School of the Primary Magisterium of Cáceres during the Second Republic, from the Bergamín Plan of I9I4, which continued those students who had already begun their studies in previous years. And who were given the opportunity to enter the like teachers through their participation in the Professional Selection Courses, following the Preparatory Plan. Who were surprised by the transition 
of curricula but did not have the title of higher bachelor required in the new, and ending with the star plan of the Second Republic, the so-called Professional Plan. We analyze the courses taught, the contents, the final revalidate of studies, the examination courts and the ways of evaluating in the Cacereña Normal School.

KEY WORDs: curriculum, primary teacher training, History of Education, Second Republic, Spain.

$\mathrm{E}$ N el momento en el que se declara la Segunda República en España, en Cáceres funcionaban dos Escuelas Normales para la formación de los maestros, una masculina y otra femenina.

El plan de estudios vigente en ese momento era el aprobado en I9I4 por el ministro de Instrucción Pública y Bellas Artes, Francisco Bergamín ${ }^{\mathrm{I}}$. Ese plan supuso un gran avance y dio una gran estabilidad a la formación de los maestros hasta la Segunda República. Entre las novedades más importantes podemos destacar las de unificar el título de maestro, suprimiendo la tradicional división de los grados elemental y superior, organizar la carrera en cuatro cursos, suprimir el «certificado de aptitud» creado a principios del siglo $\mathrm{xx}^{2}$, reducir la ratio profesor-alumno a 50, establecer el acceso a la enseñanza pública por oposición y conceder becas para aquellos alumnos aventajados que careciesen de recursos. Sin embargo, una de las características más negativas de ese plan fue el de ser esencialmente culturalista y que las disciplinas psico-socio-pedagógicas fuesen sectores marginales ${ }^{3}$.

Pocos meses más tarde del inicio de la Segunda República se aprueba la reforma más importante de la formación de los maestros en España hasta esa fecha y sin duda una de las más importantes de la historia. Es aprobada por el ministro de Instrucción Pública y Bellas Artes, Marcelino Domingo, mediante el decreto de 29 de septiembre $193 \mathrm{I}^{4}$ y complementada posteriormente con el Reglamento de las Escuelas Normales de 17 de abril de $1933^{5}$.

El Plan Bergamín fue aprobado mediante el R. D. de 30 de agosto de 1914 (Gaceta del 2 de septiembre).

2 El Real Decreto de 6 de julio de I9oo había creado los «certificados de aptitud». Art. 23: «En las Escuelas Normales se podrán dar certificados de aptitud para desempeñar Escuelas, mediante un examen de Catecismo, Lectura, Escritura, Ortografía y Aritmética».

El plan de estudios comprendía las siguientes materias: Religión y Moral, Educación física, Gramática y Literatura castellanas con ejercicios de Lectura, Caligrafía, Geografía, Historia, Matemáticas, Física y Quimica, Fisiología e Higiene, Historia Natural, Agricultura, Labores, Economía doméstica, Francés, Dibujo y Música. Y solo un poco de Pedagogía, Rudimentos de Derecho y Legislación Escolar y Prácticas de enseñanza (art. is del Plan Bergamín). Cf. Escolano Benito, A.: «Las escuelas Normales. Siglo y medio de perspectiva histórica», Revista de Educación, 269 (1982), pp. 55-76.

4 Decreto del Ministerio de Instrucción Pública y Bellas Artes de 29 de septiembre de 193 I dictando normas relativas a la preparación del Magisterio primario (Gaceta del 30 ).

5 Orden de 17 de abril de 1933 aprobando el Reglamento de Escuelas Normales (Gaceta del 4 de mayo). El Reglamento aparece completo en el Boletín Oficial del Ministerio de Instrucción Pública $y$ Bellas Artes de 4 de mayo de 1933. 
LOS PLANES DE ESTUDIOS DE LA ESCUELA NORMAL DEL MAGISTERIO PRIMARIO

DE CÁCERES EN LA SEGUNDA REPÚBLICA

FRANCISCO JAVIER ALEJO MONTES

$\mathrm{Al}$ acceder los republicanos al poder retoman el planteamiento liberal de que la igualdad de todos los ciudadanos solo se puede conseguir por medio de la educación. Fieles a este planteamiento diseñaron un ambicioso plan para erradicar el analfabetismo y para renovar la enseñanza primaria, y, para ello, consideraron que había que comenzar por la formación de los maestros ${ }^{6}$.

Por esta razón, aprueban el Decreto de 29 de septiembre para reformar las Escuelas Normales. El ministro expone en ese decreto que el primer deber de toda democracia es «resolver plenamente el problema de la instrucción pública» y hace un maravilloso panegírico del magisterio:

Urgía crear Escuelas, pero urgía más crear Maestros; urgía dotar a la Escuela de medios para que cumpliera la función social que le está encomendada, pero urgía más capacitar al Maestro para convertirlo en sacerdote de esta función; urgía elevar la jerarquía de la Escuela, pero urgía igualmente dar al Maestro de la nueva sociedad democrática la jerarquía que merece y merecerá haciéndole merecedor de ella.

Entre las principales novedades de esta reforma podemos destacar que eleva por primera vez la carrera de magisterio al mismo nivel que la mayoría de carreras universitarias (pues hasta entonces estaba en un escalafón intermedio entre la enseñanza secundaria y la universitaria $)^{7}$, establece el régimen de coeducación, impone un numerus clausus de ingreso por medio de su correspondiente examen, concede acceso directo al cuerpo de maestros nacionales a los que superasen la carrera e incorpora una reválida final para disponer el orden de promoción.

6 Cf. Ávila Fernández, A. y Holgado Barroso, J. A.: Formación del Magisterio en España. La Legislación Normalista como instrumento de poder y control (1834-2007), Madrid, Ministerio de Educación, Política Social y Deporte, 2008. Cruz Orozco, J. I.: «La política laicista de la II República: El proyecto de ley sobre los maestros laicos históricos», Historia de la Educación. Revista Interuniversitaria, 19 (2000), pp. 279-292. Molero Pintado, A.: La reforma educativa de la Segunda República Española. Primer bienio, Madrid, Santillana, 1977. Molero Pintado, A.: «Los maestros republicanos: Legislación y conflictividad profesional (I93I-I936)», Historia de la Educación. Revista Interuniversitaria, I6 (1997), pp. 285-302. Molero Pintado, A.: «La Segunda República y la formación de maestros", Tendencias Pedagógicas, i4 (2009), pp. 85-94. Pérez Galán, M.: La enseñanza en la Segunda República Española, Madrid, Edicusa, 1977. Puelles Benítez, M.: Modernidad, republicanismo y democracia: una historia de la educación en España (I898-2008), Valencia, Tirant lo Blanch, 2009. Ruiz Rodrigo, C.: «Maestro, escuela y sociedad (de la Restauración a la II República)», Historia de la Educación. Revista Interuniversitaria, I6 (1997), pp. I75-197.

7 Recordemos que la Ley Claudio Moyano la había situado en el tercer grado del escalafón superior, llamada enseñanza profesional, por debajo de la enseñanza universitaria y de la superior.

8 Cf. Alejo Montes, J.: «Aproximación histórica a la formación institucional de maestros en España», Campo Abierto, 28-i (2009), pp. I3I-I42. Alejo Montes, F. J. y Calvo Población, G. F.: «El profesorado de la Escuela Normal del Magisterio Primario de Cáceres durante la II República», en Celada Perandones, P. (ed.): Arte y Oficio de enseñar. Dos siglos de perspectiva histórica, Salamanca, Gráficas Varona, 2oII, vol. I, pp. 477-486. Ávila Fernández, A. y Holgado Barroso, J. A.: Formación del Magisterio en España. La Legislación Normalista como instrumento de poder y control (1834-2007), Madrid, Ministerio de Educación, Política Social y Deporte, 2008. Escolano Benito, A.: «Las escuelas Normales. Siglo y medio de perspectiva histórica», Revista de Educación, 269 (1982), pp. 55-76. Molero Pintado, A.: La reforma educativa de la Segunda República Española. Primer bienio, Madrid, Santillana, 1977. Molero Pintado, A.: «Los maestros republicanos: 
Además, instaura que hubiese tres fases en la formación de los maestros: una primera, de cultura general, que se cursaría en los institutos de segunda enseñanza, y exigía poseer el título de bachiller superior; la segunda fase, de formación profesional, se cursaría en las Escuelas Normales; y la tercera, de práctica docente, remunerada, se realizaría en las escuelas nacionales de la provincia.

Por todo ello, durante los primeros años de la Segunda República convivieron varios planes de estudio a la vez.

Por una parte, aquellos estudiantes que se encontraban entre segundo y cuarto curso del Plan Bergamín continuaron sus estudios hasta la extinción del plan. La posibilidad de que estos alumnos tuviesen colocación en el cuerpo del magisterio se consiguió mediante su participación en los Cursillos de selección profesional.

Por otra parte, aquellos alumnos que habían aprobado el ingreso para acceder a los estudios de ese plan de I9I4 en el curso 1931-32, y dado que no tenían el título de bachiller superior exigido en el nuevo plan republicano, estudiaron el llamado Plan preparatorio para el ingreso en el Plan profesional.

Y sin duda que el plan estrella de la Segunda República con respecto a la formación de los maestros fue el denominado Plan profesional.

\section{El Plan Preparatorio en la Escuela Normal del Magisterio Primario de Cáceres}

En septiembre de 1931 ya estaban matriculados en las dos Normales cacereñas aquellos chicos y chicas que iban a comenzar la carrera de magisterio por el plan Bergamín, que era el que estaba vigente. Al finalizar el mes se aprueba el Decreto de Reforma de las Escuelas Normales. Estos aspirantes a comenzar primero por el plan de I9I4 ya no podrán hacerlo. Tendrían que volver a hacer el examen de ingreso y previamente necesitarían haber cursado y superado los cuatro años de bachiller superior.

El ministro, consciente del problema que generaba, dispone en ese Decreto de unos artículos adicionales para realizar la transición del plan antiguo al plan moderno.

Establece que estos alumnos tenían que estudiar un plan de cultura general, similar a los estudios de bachillerato superior. Este plan se denominó Plan Preparatorio para el ingreso en el Plan Profesional.

El plan de estudios fue el siguiente: en el primer curso tenían que estudiar aritmética y álgebra, geografía (primer curso), historia (primer curso), lengua española, con ejercicios de lectura y escritura, dibujo (primer curso) y francés (primer curso).

En segundo tenían que cursar geometría y trigonometría, geografía (segundo curso), historia (segundo curso), lengua española, física, química, dibujo (segundo curso) y francés (segundo curso).

Legislación y conflictividad profesional (1931-1936)», Historia de la Educación. Revista Interuniversitaria, I6 (1997), pp. 285-302. Molero Pintado, A.: «La Segunda República y la formación de maestros», Tendencias Pedagógicas, I4 (2009), pp. 85-94. Porto Ucha, A. S.: Historias de vida. O maxisterio pontevedrés na II República, Guerra Civil e Franquismo, Pontevedra, Alén Miño, 2003. 
$\mathrm{Y}$ en el tercer curso tenían que estudiar fisiología e higiene, historia natural, agricultura, lengua y literatura, derecho y economía, labores o trabajos manuales, ejercicios físicos y música y canto.

Después de superar los tres cursos de cultura general, estos alumnos podían solicitar el ingreso en el período de formación profesional mediante el examen de ingreso y en concurrencia con el resto de los alumnos graduados de bachillerato superior.

Además de estos cursos, la Junta de Gobierno y el claustro de la Escuela Normal del Magisterio Primario de Cáceres aprobaron un cuarto curso de este Plan Preparatorio. En ese curso tuvieron que estudiar una clase diaria de pedagogía con la profesora Concepción Ruiz, una clase alterna de historia de la pedagogía con la misma profesora, otra clase alterna de composición literaria con el profesor Eduardo Málaga, dos veces por semana tuvieron una clase de legislación con el profesor Antonio Floriano Cumbreño y la asignatura de prácticas dirigida por Tomás Lucas9.

\section{Los Cursillos de selección profesional en la Escuela Normal del Magisterio Primario de Cáceres}

El Decreto de 29 de septiembre también establecía que los alumnos que ya tuviesen aprobada alguna asignatura del plan de estudios de i9I4 podían seguir sus estudios con arreglo a dicho plan, pero sin derecho a obtener colocación directa sino mediante su participación en los Cursillos de selección profesional ${ }^{\mathrm{lo}}$.

Estos cursillos comenzaron a funcionar en Cáceres el 4 de noviembre de I93I. El claustro acordó que hubiese noventa clases teóricas para cada grupo de cursillistas y que cada sesión durara aproximadamente media hora.

Y las noventa clases se repartieron de la siguiente forma: dieciséis temas de pedagogía, impartidos por los profesores Concepción García Rocasolano, García Rol y Fraile; catorce temas de idiomas, impartidos por María Esperanza Elia Pascual y Eduardo Málaga García; doce temas de matemáticas, impartidos por Evangelina Chamizo y García Rol; dieciséis temas de geografía, a cargo de María Luz Doral y los profesores Cámara y José Martínez; catorce temas de historia, impartidos por Miguel Á. Ortí Belmonte, Cámara y Tomás Lucas; diez temas de física, por Julián Rodríguez Polo y García Rol; tres temas de música, por José Gómez Crespo; tres temas de dibujo, por Tomás Lucas; y dos temas de trabajos manuales, por Concepción García Rocasolano".

9 Sesión de la Junta de Gobierno de 30 de mayo de 1934. Cf. Libro de Actas de la Junta de Gobierno de la Escuela Normal del Magisterio Primario de Cáceres, I933-1945, fol. 5.Y Sesión ordinaria de claustro de 2 de junio de 1934. Cf. Libro de Actas de Claustro de la Escuela Normal de Maestras y Mixto, I927-1943, fol. 5IV.

10 Artículo 6. ${ }^{\circ}$ adicional del Decreto de 29 de septiembre de 1931.

" Sesión ordinaria de claustro de 4 de noviembre de i93r. Cf. Libro de Actas de Claustro de la Escuela Normal de Maestras y Mixto, 1927-1943, fol. 21v.-22. 


\section{El Plan Profesional en la Escuela Normal del Magisterio Primario de Cáceres}

No cabe duda de que uno de los mejores planes de la historia de la formación de los maestros en España, si no el mejor, ha sido el republicano.

Aparte de exigirles el bachillerato superior y un año entero de prácticas, como decía anteriormente, tenían que cursar tres años en las Escuelas Normales con un plan de estudios realmente profesional, de ahí el nombre.

El plan de estudios comprendía conocimientos filosóficos, pedagógicos, sociales, metodologías especiales, materias artísticas y prácticas. Y además estaba prevista la organización de enseñanzas especiales de párvulos, retrasados, superdotados, etc., así como seminarios acerca de aquellas materias sobre las que el alumno quisiese profundizar.

En primero estudiaban elementos de filosofía, psicología, metodología de las matemáticas, metodología de la lengua y literatura españolas, metodología de las ciencias naturales y de la agricultura, música, dibujo, trabajos manuales, labores (sólo las maestras) y ampliación facultativa de idiomas.

En segundo cursaban fisiología e higiene, pedagogía, metodología de la geografía, metodología de la historia, metodología de la física y la química, música, dibujo, trabajos manuales, labores (solo las maestras) y ampliación facultativa de idiomas.

Y en el tercer curso tenían que estudiar paidología, historia de la pedagogía, organización escolar, cuestiones económicas y sociales, trabajos de seminario, trabajos de especialización y enseñanzas del hogar (solo las maestras).

\section{I. El examen de ingreso-oposición}

El tribunal encargado de seleccionar a los aspirantes lo designaba el rector de cada distrito universitario y estaba formado por un profesor/a de la Escuela Normal, un catedrático/a de instituto, un inspector/a de primera enseñanza y un maestro/a nacional ${ }^{12}$.

Pero poco duró esta composición, pues al inicio del curso 1932-33 en el claustro de la Escuela Normal del Magisterio Primario de Cáceres se leyó un telegrama del director general de Primera Enseñanza solicitando que ese tribunal quedase compuesto por tres profesores numerarios de la Escuela Normal designados por el claustro, un inspector y un maestro. Solicitaba expresamente que se excluyese del tribunal al catedrático de instituto y que si faltasen el inspector o el maestro fuesen sustituidos por otro profesor de la Escuela de Magisterio. De presidente ejercería el director de la Normal, y, en caso de no estar presente, el profesor más antiguo. Esto fue posteriormente ratificado por el Reglamento de Escuelas Normales de $1933^{13}$.

12 Artículo 5..$^{\circ}$ del Decreto de 29 de septiembre de I93I.

${ }_{13}$ El art. $6 .^{\circ}$ del Reglamento de Escuelas Normales de 1933 decía que «El Tribunal encargado de seleccionar los aspirantes a ingreso en la Normal estará formado por tres Profesoras o Profesores numerarios de Escuela Normal designados por el Claustro, un Inspector o Inspectora de Primera 
Para el curso 1932-33, la Inspección Provincial cacereña nombró para este tribunal al inspector de primera enseñanza Juvenal de Vega y Relea; el Consejo provincial de primera enseñanza de Cáceres nombró al maestro José Gabriel Sánchez Román, y el claustro de la Normal nombró a Miguel Ángel Ortí Belmonte, Julián Rodríguez Polo y a Luz Doral Pazos. Ortí Belmonte, como era el director, ejerció de presidente; Gabriel Sánchez, de secretario, y el resto, de vocales. Finalmente el claustro de la Normal autorizó al director para que "por enfermedad u otras causas justificadas» pudiese sustituir a estos profesores ${ }^{14}$.

En el curso 1933-34 el único catedrático de francés del centro exigió formar parte de este tribunal, dado que uno de los ejercicios que tenían que hacer consistía en «la traducción repentizada de un libro escrito en francés» ${ }^{15}$, pero el claustro no lo tuvo en consideración y eligió a Eladio Rodríguez (profesor numerario de paidología), Antonio Floriano Cumbreño (profesor numerario de psicología y filosofía) y Concepción Ruiz García (profesora numeraria de pedagogía $)^{16}$.

Para el curso 1934-35, la Junta de Gobierno propuso a los profesores Miguel Á. Ortí, Concepción Ruiz García y Mercedes Cantero ${ }^{17}$, pero al intentar ratificarse en el claustro hubo muchos desacuerdos y tras una breve discusión se aprobó que el tribunal fuese «designado en las vísperas de realizarse el ingreso-oposición $»^{18}$.

La Dirección General de Primera Enseñanza decidió que para ese curso 1934-35 los aspirantes solicitasen estos exámenes de ingreso durante el mes de agosto y se celebrasen a partir del día I2 de septiembre ${ }^{19}$. Por esta razón se convocó en junio un claustro para designar a los miembros del tribunal de ingreso, y después de serias discrepancias resultaron elegidos por mayoría Miguel A. Ortí, Concepción Ruiz y M. ${ }^{a}$ de las Mercedes Cantero, que a su vez habían sido los propuestos por la Junta de Gobierno ${ }^{20}$.

Para el curso 1935-36 el claustro nombró a José Martínez García, Julián Rodríguez Polo y Concepción Ruiz García, los cuales, según el secretario del claustro, habían sido los «propuestos por la Junta de Gobierno»"21, aunque en las actas de la Junta de Gobierno no aparecen.

enseñanza designado por el Consejo de Inspección y un Maestro o Maestra nacional de la localidad, designado por el Consejo provincial de Protección escolar. Si entre los Profesores designados para formar parte del Tribunal figurase el Director de la Escuela, será éste quien presida. En otro caso ocupará la Presidencia el Profesor de Normal que posea más antigüedad».

${ }_{14}$ Sesión ordinaria de claustro de 14 de septiembre de 1932. Cf. Libro de Actas de Claustro de la Escuela Normal de Maestras y Mixto, 1927-1943, fols. 30v.-31.

is Sesión de claustro de I2 de junio de I933. Cf. ibidem, fol. 44v.

16 Sesión de claustro de 24 de junio de 1933 . Cf. ibidem, fol. $44 \mathrm{v}$.

${ }_{17}$ Sesión de la Junta de Gobierno de 30 de mayo de i934. Cf. Libro de Actas de la Junta de Gobierno de la Escuela Normal del Magisterio Primario de Cáceres, 1933-1945, fol. 5v.

18 Sesión de claustro de 2 de junio de 1934. Cf. Libro de Actas de Claustro de la Escuela Normal de Maestras y Mixto, 1927-1943, fol. 52.

19 Orden de la Dirección general de Primera enseñanza de 7 de junio de 1934 (inserta en la Gaceta del 8).

2o Sesión de claustro de I2 de junio de i934. Cf. ibidem, fols. 52-53.

${ }_{21}$ Sesión de claustro de I de julio de 1935. Cf. ibidem, fol. 64 . 
Finalmente, para el curso 1936-37 el claustro acordó por unanimidad que el tribunal de ingreso lo formasen los profesores Eladio Rodríguez Gallego, María Esperanza Elía Pascual y Antonio Floriano Cumbreño, «en unión del Inspector y el Maestro que designen respectivamente la Junta de Inspectores y el Consejo Provincial de r. ${ }^{a}$ Enseñanza» ${ }^{22}$.

Pero para ese curso 1936-37, y por supuesto, los siguientes, el examen de ingreso ya no se celebró, pues tras el inicio de la Guerra Civil, la Junta de Defensa Nacional acordó dejar en suspenso hasta nueva orden dicho examen de ingreso en el grado profesional del Magisterio ${ }^{23}$.

\subsection{La reválida final de estudios}

Al terminar el tercer curso los alumnos tenían que hacer un examen de conjunto que serviría «para determinar el orden de prelación entre ellos a los efectos de su colocación provisional en el período de práctica docente». El tribunal encargado de hacer este examen lo designaba el rector de la Universidad y estaba compuesto por un catedrático de universidad, tres profesores de la Escuela Normal y un inspector de Primera Enseñanza ${ }^{24}$.

Dado que la primera promoción del Plan Profesional de Cáceres finalizó sus estudios en el curso 1933-34, a finales de ese curso la Junta de Gobierno acordó proponer al rector de la Universidad de Salamanca a los profesores Antonio Floriano, Eduardo Málaga y Carmen Queimadelos para formar parte de este tribunal de reválida ${ }^{25}$, aunque posteriormente, por razones que desconozco, la Junta de Gobierno celebrada en junio propuso a los tres candidatos anteriores y a José Martínez, que ejercería de presidente, para formar parte de este tribunal ${ }^{26}$, lo que me hace sospechar que el catedrático de universidad no participó.

Varios profesores del claustro solicitaron «benevolencia del tribunal calificador de la reválida», basándose en la irregularidad con que a su juicio se había desenvuelto el plan de estudios, y además, reclamaron que se les diese «el cuestionario con ocho días de anticipación a los exámenes», pero, tras leer el secretario la Orden de 3I de mayo de 1932, que regulaba los exámenes de los planes Preparatorio y Profesional ${ }^{27}$, y como en esos momentos se desconocía aún la composición de dicho tribunal, el claustro acordó que no podía aún saberse «ni anticiparse

22 Sesión de claustro de i8 de junio de 1936. Cf. ibidem, fols. 74-75.

23 Boletín Oficial de la Junta de Defensa Nacional en España. Burgos, 29 de agosto de 1936.

24 Artículo i3 del Decreto 29 de septiembre de 1931.

${ }_{25}$ Sesión de la Junta de Gobierno de 7 de abril de 1934. Cf. Libro de Actas de la Junta de Gobierno de la Escuela Normal del Magisterio Primario de Cáceres, 1933-1945, fol. $5 \mathrm{v}$.

${ }_{26}$ Sesión de la Junta de Gobierno de 4 de junio de 1934. Cf. ibidem, fol. 6v. Y sesión extraordinaria de claustro de 12 de junio de 1934. Cf. Libro de Actas de Claustro de la Escuela Normal de Maestras y Mixto, 1927-1943, fol. 53.

${ }_{27}$ Orden de 3I de mayo de 1932 (Gaceta del 4 de junio) de la Dirección General de Primera Enseñanza dando disposiciones para proporcionar efectividad al espíritu de la reforma de las Normales y para unificar la labor de los claustros en orden a los exámenes. 
absolutamente nada acerca del citado cuestionario» y que todas las observaciones pertinentes se hiciesen ante el tribunal que se formase para esta cuestión ${ }^{28}$.

Para el curso 1935-36 el rector de la Universidad salmantina solicitó a la Normal cacereña que se nombrasen cuatro profesores numerarios y un inspector de Primera Enseñanza para constituir el tribunal. La Junta de Gobierno nombró como presidente a Miguel Á. Ortí Belmonte y como vocales a las profesoras Evangelina Chamizo, Concepción Ruiz y Mercedes Cantero. Por su parte, la Inspección de Cáceres nombró al inspector Lucas García Rol'29.

Para el curso i936-37 el claustro nombró a los profesores Miguel Á. Ortí Belmonte, Evangelina Chamizo González, Antonio Floriano Cumbreño y M. ${ }^{a}$ de las Mercedes Cantero Roncero ${ }^{30}$, aunque esta última profesora solicitó que se le relevase «de formar parte de este tribunal» por razones personales, por lo que el claustro acordó "por unanimidad conceder un voto de confianza al Sr. Director» para que, en caso necesario y justificado, pudiese sustituir a cualquiera de los profesores de este tribunal ${ }^{3 \mathrm{I}}$.

\subsection{Trabajos de seminario y trabajos de especialización}

En el tercer curso de este Plan Profesional los alumnos tenían que cursar «trabajos de seminario» $\mathrm{y}$ «trabajos de especialización».

Los «trabajos de seminario» estaban encaminados a «orientar el trabajo personal de los alumnos de modo que pudiesen intensificar sus estudios en una dirección acorde con su particular disposición, a fin de ensanchar el horizonte cultural del Magisterio primario»32.

En la Escuela Normal de Cáceres estos trabajos comenzaron en el curso I93334. Tras muchas deliberaciones, el claustro acordó que para ese curso se impartieran cuatro seminarios: Investigación psicológica escolar, a cargo del profesor de psicología Antonio Floriano y del profesor de paidología Eladio Rodríguez; Investigación histórica, a cargo del profesor de historia Miguel Á. Ortí Belmonte; Monografías geográficas, a cargo del profesor de geografía José Martínez y de la profesora de matemáticas Evangelina Chamizo; y Comprobación de técnicas psicológicas, a cargo de la profesora de pedagogía Concepción Ruiz ${ }^{33}$.

Para el curso 1934-35 la Junta de Gobierno y el claustro aprobaron Monografías históricas, dirigidas por Miguel Á. Ortí Belmonte; Monografías geográficas,

28 Claustro de 27 de abril de 1934. Cf. Libro de Actas de Claustro de la Escuela Normal de Maestras y Mixto, 1927-1943, fol. 50.

29 Sesión de la Junta de Gobierno de 3i de mayo de i935. Cf. Libro de Actas de la Junta de Gobierno de la Escuela Normal del Magisterio Primario de Cáceres, 1933-1945, fol. I3v.

30 Sesión ordinaria de claustro de 26 de mayo de 1936. Cf. Libro de Actas de Claustro de la Escuela Normal de Maestras y Mixto, 1927-1943, fol. 72.

${ }_{31}$ Sesión de claustro de 3 de junio de 1936. Cf. ibidem, fol. $73 \mathrm{v}$.

32 Artículo 8..$^{\circ}$ del Decreto de 29 de septiembre de r931.

33 Sesión ordinaria de claustro de 5 de junio de 1933. Cf. Libro de Actas de Claustro de la Escuela Normal de Maestras y Mixto, 1927-1943, fols. 43v.-44. 
por la profesora María Luz Doral; Investigaciones psicológicas escolares, por los profesores Concepción Ruiz García y C. Antonio Floriano Cumbreño, y Literatura regional, por Eduardo Málaga ${ }^{34}$.

Para el curso 1935-36 se aprobaron solamente dos: Monografías históricas, dirigidas por Miguel Á. Ortí Belmonte, e Investigaciones psicológicas escolares, por los profesores Ruiz García y Floriano Cumbreño. El profesor de música Gómez Crespo solicitó descargar de estos trabajos a los alumnos, pero el director respondió que no era posible por ser reglamentarios ${ }^{35}$.

Para el aciago curso 1936-37 el claustro aprobó otros dos trabajos de seminario: Monografías históricas, dirigidas por el profesor Miguel Á. Ortí Belmonte, e Investigaciones psicológicas y paidológicas, por los profesores Eladio Rodríguez, Antonio Floriano Cumbreño y Concepción Ruiz García ${ }^{36}$.

Con respecto a los «trabajos de especialización» se pretendía que los alumnos se especializasen, de la forma que ellos estimasen conveniente, entre enseñanzas especiales de párvulos, retrasados, superdotados, etc. ${ }^{37}$.

El claustro de la Normal de Cáceres aprobó para el curso 1933-34 los siguientes «trabajos de especialización»: Agrimensura, impartido por la profesora Evangelina Chamizo; Cosmografía y Meteorología, impartido por el profesor Eladio Rodríguez; Estudio de los tintes en los diversos tejidos, impartido por la profesora Mercedes Cantero; Industrias agrícolas de la región: avicultura, apicultura, cunicultura y sericicultura, impartido por la profesora María del Carmen Queimadelos; Enseñanza de superdotados y de retrasados, impartido por la profesora Concepción Ruiz; y estaban, además, las especializaciones obligadas de pároulos y de prácticas agrícolas. Además, acordaron en el claustro, que «se organizarán ocasionalmente visitas a Museos, excursiones, viajes instructivos y Misiones pedagógicas, solicitando al efecto las subvenciones precisas $\gg^{38}$.

Para el curso 1934-35 se aprobaron solamente dos: Agricultura, obligatoria para los alumnos varones, dirigida por la profesora María del Carmen Queimadelos; y Pároulos, obligatoria para las alumnas, bajo la dirección de los profesores Concepción Ruiz García y C. Antonio Floriano Cumbreño ${ }^{39}$.

Para el curso 1935-36 el claustro ratificó los tres siguientes: Agricultura: Prácticas agrícolas, obligatorias para los alumnos, dirigidas por la profesora María del Carmen Queimadelos Viéitez; Pároulos, obligatoria para las alumnas, bajo la

34 Sesión de la Junta de Gobierno de 30 de mayo de 1934. Cf. Libro de Actas de la Junta de Gobierno de la Escuela Normal del Magisterio Primario de Cáceres, I933-1945, fol. 5r.-v. Y Sesión ordinaria de claustro de 2 de junio de 1934. Cf. Libro de Actas de Claustro de la Escuela Normal de Maestras y Mixto, 1927-1943, fol. 5Iv.

35 Sesión ordinaria de claustro de 22 de junio de 1935 . Cf. ibidem, fol. 63v.

36 Sesión de claustro de 3 de junio de i936. Cf. ibidem, fol. 73.

37 Artículo 9. ${ }^{\circ}$ del Decreto de 29 de septiembre de 1931.

${ }_{38}$ Sesión ordinaria de claustro de 5 de junio de 1933. Cf. Libro de Actas de Claustro de la Escuela Normal de Maestras y Mixto, I927-1943, fols. 43v.-44.

39 Sesión de la Junta de Gobierno de 30 de mayo de 1934. Cf. Libro de Actas de la Junta de Gobierno de la Escuela Normal del Magisterio Primario de Cáceres, 1933-1945, fol. 5r.-v. Y Sesión ordinaria de claustro de 2 de junio de 1934. Cf. Libro de Actas de Claustro de la Escuela Normal de Maestras y Mixto, 1927-1943, fol. 5Iv. 
dirección de los profesores Concepción Ruiz García y Antonio Floriano Cumbreño; y Pedagogía, a cargo de los tres profesores de la sección, Eladio Rodríguez Gallego, Concepción Ruiz García y Antonio Floriano Cumbreño ${ }^{40}$.

Y para el curso 1936-37 el claustro aprobó tres trabajos de especialización: Prácticas agrícolas, obligatorias para los alumnos varones, dirigidas por la profesora de metodología de las ciencias naturales y de la agricultura, María del Carmen Queimadelos Viéitez; Párvulos, obligatoria para las alumnas, bajo la dirección de los profesores Antonio Floriano Cumbreño y Concepción Ruiz García; y Pedagogía, a cargo de Eladio Rodríguez Gallego, Antonio Floriano Cumbreño y Concepción Ruiz García $a^{45}$.

Pero en el curso 1936-37 hubo problemas con estos trabajos de seminario y con los de especialización, pues se suprimió la coeducación, a las chicas se les permitió continuar con los estudios, pero se suspendió la docencia masculina ${ }^{42}$, dado que llamaron a filas a los mozos de los reemplazos de 1933, 1934 y $1935^{43}$.

\section{Los cursos impartidos en la Escuela Normal del Magisterio Primario de Cáceres durante la Segunda República}

En la Escuela Normal del Magisterio Primario de Cáceres funcionaron en el curso 1931-32 tres cursos del Plan Bergamín de 1914 (segundo, tercero y cuarto), un curso del Plan Preparatorio, otro del Plan Profesional y los cursillos de selección profesional.

Como el Decreto de Reforma de las Escuelas Normales se publicó el 30 de septiembre de 1931, en Cáceres fue necesario retrasar el inicio del curso 193I-32.

El claustro se reunió en octubre de 1931 y decidió anunciar en los periódicos locales, «para mayor divulgación», la matrícula de los alumnos del Plan Bergamín y dejar en suspenso la matrícula del curso del Plan Preparatorio. Además, resolvió solicitar a la Superioridad que permitiese matricularse en segundo del Plan Bergamin a aquellos alumnos a los que les faltase una o dos asignaturas de primero, condicionando esa matrícula a aprobar esas asignaturas en junio «y siempre, claro es, que la aprobación en mayo de las asignaturas del $2 .^{\circ}$ curso sea a base de que en junio aprueben las que signifiquen precedencia»4 .

Un nuevo claustro celebrado en noviembre aprobó el comienzo de las clases de primero del Plan Preparatorio y de segundo del Plan Bergamín para el día 9 de noviembre de $193 \mathrm{I}^{45}$, dejando en suspenso las del Plan Profesional.

40 Sesión de claustro de 22 de junio de 1935. Cf. ibidem, fol. 63v.

${ }_{41}$ Sesión de claustro de 3 de junio de 1936. Cf. ibidem, fol. 73.

${ }_{42}$ Orden de 22 de septiembre de 1936. Boletín Oficial de la Junta de Defensa Nacional de Espa$\tilde{n} a$, núm. 28, de 25/09/1936, p. II2.

43 Decreto n. ${ }^{\circ} 29$ de 8 de agosto de 1936 disponiendo la urgente incorporación a filas (Boletín Oficial de la Junta de Defensa Nacional de España de 9 de agosto de 1936).

${ }_{44}$ Sesión de claustro de 2 de octubre de 193I. Cf. Libro de Actas de Claustro de la Escuela Normal de Maestras y Mixto, 1927-1943, fol. 19 .

45 Sesión ordinaria de claustro de 2 de noviembre de 193I. Cf. ibidem, fol. 20. 
Y por fin, el curso del Plan Profesional comenzó definitivamente el iz de enero de 1932 por decisión del claustro «utilizando si necesario es en algunas horas el salón de biblioteca hasta que terminen los cursillos y se pueda disponer del aula en que se celebran éstos» ${ }^{46}$.

Y dado que había varios alumnos del grado profesional que a la vez eran cursillistas y no podían asistir a los dos sitios a la vez, acordaron consultar si se les podría dispensar de la asistencia a las clases durante el tiempo que durasen los cursillos «y a reserva de que a fin de curso se les someta a un examen general en todas las materias y para apreciar su cultura en sí misma y en relación con los demás alumnos de este curso» ${ }^{47}$.

Como las clases de ese curso 1931-32 comenzaron más tarde, el claustro se planteó finalizarlo también más tarde, pero decidió finalmente que los cursos de los planes Preparatorio y Bergamín no se prorrogasen porque comenzaron con sólo un mes de retraso «y ello se ha tenido en cuenta en las tareas escolares, que no se han interrumpido por huelgas ni ninguna otra causa, aparte que sería materialmente imposible la permanencia de todos los alumnos oficiales mientras los exámenes de los alumnos libres».

Sin embargo, para los alumnos del Plan Profesional se decidió que el curso se prorrogase hasta el día io de junio «y no más por innecesario y para que los profesores queden libres para acudir al cursillo proyectado en Madrid para el 20 de junio» ${ }^{8}$.

Finalmente, la Dirección General de Primera Enseñanza confirmó que el curso del Plan Profesional se prorrogase hasta el to de junio, pero también exigió lo mismo para el del Plan Preparatorio ${ }^{49}$.

En el curso 1932-33 se impartieron tercero y cuarto del Plan Bergamín, segundo del Plan Preparatorio y primero y segundo del Plan Profesional.

En el curso 1933-34 se impartió cuarto del Plan Bergamín, tercero del Plan Preparatorio y los tres cursos del Plan Profesional.

En el curso 1934-35 se impartió cuarto del Plan Preparatorio y los tres cursos del Plan Profesional.

En el curso 1935-36 ya solo se impartió el Plan Profesional.

Pero a partir del curso 1936-37 hubo graves problemas para impartir la docencia de forma normal, pues a la depuración de maestros en la provincia de Cáceres (que no solo abarcó a los maestros en propiedad, sino también a los alumnos de la Escuela Normal, como demuestran Juan Carlos Vázquez y Santiago García) ${ }^{\text {s。, }}$, hay que añadir que se suprimió la coeducación y que se suspendieron las clases masculinas porque los chicos fueron llamados a filas.

46 Sesión ordinaria de claustro de ir de enero de 1932. Cf. ibidem, fol. 24.

47 Sesión ordinaria de claustro de II de enero de 1932. Cf. ibidem, fol. 24.

${ }_{48}$ Sesión ordinaria de claustro de 9 de mayo de 1932 . Cf. ibidem, fol. $28 \mathrm{v}$.

49 Orden del 31 de mayo de 1932 (Gaceta del 4 de junio) de la Dirección General de Primera Enseñanza.

5o Vázquez Calvo, J. C. y García Jiménez, S.: La depuración de la enseñanza primaria en la provincia de Cáceres, I936-1944. El personal docente, la administración educativa y la organización escolar, Cáceres, Institución Cultural El Brocense y Excma. Diputación Provincial de Cáceres, 2008. 
FRANCISCO JAVIER ALEJO MONTES

Además, en septiembre de 1936 el edificio recién creado por la Segunda República para la formación de los maestros cacereños se destinó a hospital militar para los heridos en el frente, por lo que se tuvieron que trasladar las clases de las chicas al Ateneo y al Colegio de Veterinaria de Cáceres.

$\mathrm{Y}$, finalmente, esas mismas chicas, al mando de la profesora de labores de la Normal, fueron requeridas para confeccionar prendas militares para el ejército.

\section{Las formas de evaluar en los distintos planes de estudios de la Escuela Normal del Magisterio Primario de Cáceres}

Dado que en el curso I93I-32 funcionaban a la vez muchos cursos y de varios planes distintos, el claustro cacereño se reunió en abril para decidir los sistemas de evaluación de cada plan de estudios.

Con respecto al Plan Bergamín decidió que cada profesor calificase a sus alumnos por las notas que tuviese «de su aprovechamiento durante el curso, sin perjuicio de que si algún profesor lo cree necesario complete los elementos de juicio con ejercicios escritos, problemas, etc., que encargue a sus alumnos».

Para juzgar a los alumnos del Plan Preparatorio el claustro acordó que cada profesor exigiese las pruebas que creyese «precisas para juzgar con acierto a los alumnos en su respectiva materia».

Y con respecto al Plan Profesional acordaron evaluar conforme al Decreto de Reforma de las Escuelas Normales ${ }^{51}$, el cual establecía que el claustro tenía que determinar al final del curso las listas de los alumnos que por su trabajo y preparación se hallasen en condiciones de pasar al curso siguiente. Y si lo estimaba necesario tenía que organizar pruebas escritas evaluadas por un tribunal para aquellos alumnos cuya calificación pudiese ofrecer dudas a los profesores. Y si no superaban estas pruebas tenían que repetir curso $^{52}$.

El claustro cacereño acordó en 1932 que se hiciese la lista por orden de méritos de los alumnos del grado profesional que podían pasar al segundo curso "previo informe de los profesores que explican las materias de dicho curso y como consecuencia de la labor realizada por dichos alumnos, a los que no procede, por ello, dar papeleta de examen» ${ }^{53}$.

Sin embargo, para unificar la labor de los claustros con respecto a estos sistemas de evaluación, el director general de Primera Enseñanza, Rodolfo Llopis, publicó una Orden el 3i de mayo de 1932 en la que establecía cómo debían de formarse las comisiones calificadoras de los planes de estudio Preparatorio y Profesional.

Con respecto al Plan Preparatorio consideraba necesario que las comisiones aprobasen o suspendiesen por unanimidad. De no existir esta unanimidad, el

sI Sesión ordinaria de 25 de abril de 1932. Cf. Libro de Actas de Claustro de la Escuela Normal de Maestras y Mixto, 1927-1943, fol. 27v.

$52 \quad$ Artículo I2 del Decreto de 29 de septiembre de r93I.

53 Sesión ordinaria de claustro de 9 de mayo de 1932. Libro de Actas de Claustro de la Escuela Normal de Maestras y Mixto, 1927-1943, fol. 29r.-v. 
alumno/a en cuestión tendría que realizar un examen de conjunto en el mes de junio. Si lo suspendiese lo repetiría en septiembre y de volverlo a suspender tendría que realizar nuevas pruebas en futuras convocatorias «ya que no habrá en lo sucesivo matrícula oficial para el primer curso de Cultura general».

Esta comisión calificadora tenía que hacer, finalmente, una lista de mérito con los alumnos que lograsen pasar al curso siguiente, bien por aprobación unánime de los profesores, bien por el resultado de las pruebas de conjunto de junio o septiembre.

Esta Orden también permitió (con arreglo al artículo r. ${ }^{\circ}$ adicional del Decreto de Reforma de las Escuelas Normales de 29 de septiembre de 193I) a los alumnos que hubiesen aprobado el antiguo examen de ingreso examinarse en junio o septiembre del primer curso de Cultura general, sometiéndose a las mismas pruebas de conjunto que los alumnos oficiales.

Los tribunales para juzgar estos exámenes estaban constituidos por tres profesores de distintos grupos de materias designados por el claustro.

Por otra parte, los alumnos del Plan Profesional tenían que ser calificados o examinados de la misma forma que los alumnos del Plan Preparatorio. Si no aprobaban tenían que repetir curso como alumnos oficiales. Los que aprobaban pasaban de curso y se realizaba una lista de mérito con el mismo criterio que los alumnos del plan de cultura generals4.

Como consecuencia de esta Orden de Rodolfo Llopis el claustro cacereño se reunió a principios de junio para nombrar las distintas comisiones calificadoras.

Con respecto al Plan Preparatorio acordó que para los alumnos oficiales esta comisión estuviese formada por los profesores Miguel Á. Ortí, Eladio Rodríguez, M. ${ }^{a}$ Esperanza Elia, M. ${ }^{a}$ Luz Doral, Gustavo Hurtado y Acisclo Benabal. Y para los alumnos libres, por Miguel Á. Ortí, Eladio Rodríguez y María Luz Doral. Y convinieron que «cada profesor, al calificar a los alumnos oficiales, adjudiquen puntos de cero a diez, y para calificar a los alumnos libres de cero a veinte, con el fin de que haya la conveniente uniformidad de criterio en dichas calificaciones».

Con respecto al Plan Profesional, acordaron que la comisión calificadora de los alumnos del primer curso estuviese formada por los profesores de dicho curso Eladio Rodríguez (que ejercería de presidente), María del Carmen Queimadelos, Concepción Ruiz, Concepción García, Antonio Floriano, Eduardo Málaga, Gustavo Hurtado, José Gómez Crespo y Acisclo Benabal, «y quedó convenido que cada profesor adjudique puntos de cero a diez»"s.

En el curso 1932-33 se presentó el problema de dos alumnos del Plan Profesional que habían interrumpido sus estudios, uno para prestar sus deberes militares y otra por enfermedad, ambos habían pagado su matrícula y sus derechos de examen antes de conocerse el Reglamento de Escuelas Normales, que dice que

${ }_{54}$ Orden de 3r de mayo de 1932 (Gaceta del 4 de junio) de la Dirección General de Primera Enseñanza dando disposiciones para proporcionar efectividad al espíritu de la reforma de las Normales y para unificar la labor de los claustros en orden a los exámenes.

"Sesión ordinaria de claustro de 7 de junio de 1932. Cf. Libro de Actas de Claustro de la Escuela Normal de Maestras y Mixto, 1927-1943, fols. 29v.-30. 
aquellos alumnos que figurasen en las listas de aprobados en el ingreso de las Escuelas Normales o en las de mérito relativo de cualquiera de los cursos «y que por causa justificada, a juicio del claustro de profesores, se vean precisados a interrumpir sus estudios», podrán matricularse para continuar en el curso siguiente, pero "perderán el número que hubiesen obtenido en el curso en que interrumpieron sus estudios y serán colocados al final de la lista de mérito relativo correspondiente al curso a que se incorporen». Y los alumnos que fuesen llamados al servicio militar «tendrán derecho a ocupar de nuevo plaza en las condiciones del apartado anterior, al obtener licencia, sea cualquiera la duración del tiempo que permanezcan en filas» ${ }^{56}$.

El claustro se reunió en septiembre de 1933 y, «teniendo en cuenta que las leyes no tienen efecto retroactivo, y habida cuenta de la Orden de 3I de mayo de 1932», acordó conceder examen a estos alumnos en la convocatoria de septiembre de $1933^{57}$.

El curso 1933-34 era el último en el que se podía cursar el Plan de I9I4. Para ir liquidando ese plan, el director general de Primera Enseñanza aprobó una orden autorizando a matricularse oficialmente en el cuarto curso de ese plan a todos aquellos alumnos que tuviesen suspenso el segundo curso de Música o asignaturas del tercer curso, aunque «no podrá darse validez a la aprobación de las del cuarto año si previamente no han aprobado las de segundo y tercero» ${ }^{8}$. Los alumnos de la Normal cacereña implicados se quejaron amargamente de esta orden. El alumno representante en claustro expresó que sus compañeros habían enviado «una instancia al Ministerio solicitando la anulación de mencionada Orden o rogando que dicha disposición se considere en vigor solamente para las asignaturas incompatibles» $\$$.

Con respecto al Plan Profesional, en ese mismo curso 1933-34, en Junta de Gobierno se acordó demorar las calificaciones trimestrales del grado profesional hasta que se pudiesen hacer las del segundo trimestre con las del tercero, "porque las del 2. ${ }^{\circ}$ trimestre serían ahora sin bastantes elementos de juicio» ${ }^{60}$.

Y, además, los alumnos se quejaron de falta de imparcialidad por parte de los profesores en las calificaciones trimestrales. El representante en el claustro del primer curso profesional, Samuel Martín Retortillo, expuso en un claustro, en nombre de sus compañeros, que «a su juicio hubo alguna parcialidad en las primeras calificaciones trimestrales del curso actual, rogando se corrija en los restantes trimestres y amenazando que en caso contrario emprenderán el camino que debieran seguir». Estas palabras produjeron un gran disgusto en el profesorado,

56 Artículo io del Reglamento de Escuelas Normales de 1933.

57 Sesión ordinaria de claustro de 25 de septiembre de 1933. Cf. Libro de Actas de Claustro de la Escuela Normal de Maestras y Mixto, 1927-1943, fol. 46.

${ }_{58}$ Orden de la Dirección General de Primera Enseñanza de 20 de octubre de 1933 (Gaceta del 29).

59 Claustro de 27 de abril de 1934. Cf. Libro de Actas de Claustro de la Escuela Normal de Maestras y Mixto, 1927-1943, fol. 49 .

60 Sesión de la Junta de Gobierno de 7 de abril de 1934. Cf. Libro de Actas de la Junta de Gobierno de la Escuela Normal del Magisterio Primario de Cáceres, 1933-1945, fol. $4 \mathrm{v}$. 
y el director, en nombre del mismo, leyó los artículos del Reglamento referentes al modo de calificar a los alumnos del nuevo plan, demostrando al Sr. Martín Retortillo «que no ha habido parcialidad ni padrinazgo de ningún género en dichas calificaciones, pues ello sería indigno en el profesorado de este Centro, y rechazando, en fin, por improcedentes las palabras del referido alumno» ${ }^{6 \mathrm{r}}$.

En el curso 1934-35 el claustro acordó que los exámenes extraordinarios de enero se realizasen en la segunda quincena, en el día que fijase el director ${ }^{62}$.

Para ese curso la Junta de Gobierno nombró como tribunal para juzgar a los distintos cursos del Plan Preparatorio a los profesores Eladio Rodríguez Gallego, Antonio Floriano Cumbreño y Concepción Ruiz García ${ }^{63}$. El claustro, además, acordó "calificar en conjunto» a los alumnos oficiales del cuarto curso del Plan Preparatorio y a los del Plan Profesional y que las actas se cerrasen el 25 de mayo "por ser feriados los restantes días» ${ }^{64}$. Por su parte, la Junta de Gobierno acordó (y posteriormente fue ratificado por el claustro) que aquellos alumnos cuya puntuación media aritmética no superase los cinco puntos tenían que ser examinados por los profesores correspondientes el día 5 de junio, previa petición de los interesados 65 .

Para el curso 1935-36 se acordó que la calificación del segundo trimestre se hiciese el día i de abril, para lo cual se reunirían todos los profesores y entregarían por escrito las oportunas calificaciones al secretario, quien haría «el cómputo y promedio para la lista de méritos de los alumnos en presencia del Sr. Director y de los demás profesores de cada curso, inutilizando después las notas entregadas por el profesorado, una vez firmadas las actas». Así se acordó unánimemente, $y$ que si algún profesor no quisiera entregar sus notas, se hiciese «la lista de mérito prescindiendo de ellas y hallando el promedio con las notas recibidas» ${ }^{66}$.

En una sesión de claustro celebrada en marzo de 1936 la profesora Evangelina Chamizo propuso que se solicitara a la Superioridad otra forma más adecuada de calificar a los alumnos "para que no se dé el caso de que puedan aprobar algunas materias sin el estudio correspondiente». El claustro aceptó la proposición por unanimidad y decidió comunicárselo al inspector general cuando realizase «la visita a este Centro» ${ }^{67}$.

${ }^{61}$ Sesión de claustro de 27 de abril de 1934. Cf. Libro de Actas de Claustro de la Escuela Normal de Maestras y Mixto, 1927-1943, fol. 49r.-v.

62 Sesión ordinaria de claustro de ro de enero de 1935. Cf. ibidem, fol. $56 \mathrm{v}$.

63 Sesión de Junta de Gobierno de 4 de mayo de 1935. Cf. Libro de Actas de la Junta de Gobierno de la Escuela Normal del Magisterio Primario de Cáceres, I933-1945, fol. I2v.

${ }^{6} 4$ Sesión ordinaria de claustro de 16 de mayo de 1935 . Cf. Libro de Actas de Claustro de la Escuela Normal de Maestras y Mixto, 1927-1943, fol. 60.

65 Acuerdo tomado en la sesión de Junta de Gobierno de 31 de mayo de 1935. Cf. Libro de Actas de la Junta de Gobierno de la Escuela Normal del Magisterio Primario de Cáceres, 1933-1945, fol. 13v. Posteriormente, fue ratificado en la sesión ordinaria de claustro de 31 de mayo de 1935. Cf. Libro de Actas de Claustro de la Escuela Normal de Maestras y Mixto, 1927-1943, fol. 63.

${ }_{66}$ Sesión ordinaria de claustro de 14 de marzo de 1936. Cf. ibidem, fol. 69.

${ }_{67}$ Sesión ordinaria de claustro de i4 de marzo de 1936. Cf. ibidem, fol. 69. 


\section{Conclusión}

Con la Segunda República, Cáceres, una ciudad pequeña y tranquila, vivió una gran revolución en sus únicos centros de educación superior que tenía, que eran las dos Escuelas Normales masculina y femenina.

Estas cambiaron radicalmente su composición, se unificaron y se convirtieron en estudios universitarios, los primeros que tuvo esta localidad extremeña.

El Ayuntamiento y la Diputación participaron activamente sufragando parte de la financiación del nuevo edificio destinado a la formación de los maestros.

El claustro de la Normal cacereña expresó su satisfacción, y así se lo transmitió al ministro de Instrucción Pública y al director general de Primera Enseñanza, por la excelente reforma para la formación de los maestros emprendida por los dirigentes republicanos, por entender que ganaría mucho la cultura y el prestigio del Magisterio Primario y, a la vez, porque elevaría el nivel docente y social de los maestros.

La Segunda República ideó un plan profesional extraordinario para su formación, el mejor hasta la fecha, pues todos habían sido planes culturalistas, y uno de los mejores de la historia, si no el mejor.

Para ponerlo en marcha fue necesario compatibilizar una serie de planes de estudio que funcionaron de forma independiente.

Cuando se aprobó el nuevo plan de estudios de 1931 ya estaban matriculados los alumnos en primero del Plan Bergamín de i9i4. Tenían su derecho a estudiar magisterio, pero no cumplían el principal requisito para el nuevo plan, que era tener el título de bachiller superior. Por ello, a estos estudiantes se les derivó hacia el llamado Plan Preparatorio para el posterior ingreso en el Plan Profesional, que consistía básicamente en realizar unos estudios similares a los del bachillerato. El plan de estudios era inicialmente de tres años, pero en Cáceres se aumentó a cuatro.

A los alumnos del Plan Bergamín que ya tenían algún curso aprobado se les permitió continuar sus estudios, pero no tendrían acceso a la enseñanza pública, al igual que les sucedía a todos los titulados en cursos anteriores que no hubiesen aprobado previamente la oposición.

Para solucionar ese problema, el Ministerio ideó los Cursillos de selección profesional. En Cáceres comenzaron a funcionar en noviembre de 1931. Presento las asignaturas y los profesores que los impartieron.

Sin duda que el plan estrella fue el denominado Plan Profesional. Se llamó así porque se quería que en la Normal se aprendiese realmente la profesión, no la cultura necesaria que debería poseer un maestro. Por ello, se les exigió previamente tener el bachillerato superior.

Se recoge la formación y composición del examen de ingreso-oposición, de la reválida fin de estudios, así como las formas de evaluar en los distintos planes de estudio en Cáceres. También se recogen los trabajos de seminario y de especialización que se impartieron en los distintos años de la Segunda República en la Normal cacereña. 
Pero con el inicio de la Guerra Civil se suprimió la coeducación, se les quitó el edificio para convertirlo en hospital de los heridos en el frente y comenzó la depuración en la provincia de Cáceres, no solo de los docentes en ejercicio, sino también de los alumnos normalistas.

Se suspendieron las clases para los chicos, dado que fueron llamados a filas, mientras que las chicas se tuvieron que trasladar a otros sitios que no reunían las mínimas condiciones exigidas, como el Ateneo y el Colegio de Veterinaria de Cáceres, además de que se las requirió para que, junto a la profesora de Labores, confeccionasen prendas militares para el ejército.

Todo ese cúmulo de despropósitos hizo que, tras el inicio de la Guerra Civil, los estudios presenciales de la Escuela Normal del Magisterio Primario de Cáceres quedasen en suspenso. 